\title{
Clifford Fibrations and Possible Kinematics
}

\author{
Alan S. MCRAE \\ Department of Mathematics, Washington and Lee University, Lexington, VA 24450-0303, USA \\ E-mail:mcraea@wlu.edu
}

Received April 10, 2009, in final form June 19, 2009; Published online July 14, 2009

doi:10.3842/SIGMA.2009.072

\begin{abstract}
Following Herranz and Santander [Herranz F.J., Santander M., Mem. Real Acad. Cienc. Exact. Fis. Natur. Madrid 32 (1998), 59-84, physics/9702030] we will construct homogeneous spaces based on possible kinematical algebras and groups [Bacry H., Levy-Leblond J.-M., J. Math. Phys. 9 (1967), 1605-1614] and their contractions for 2dimensional spacetimes. Our construction is different in that it is based on a generalized Clifford fibration: Following Penrose [Penrose R., Alfred A. Knopf, Inc., New York, 2005] we will call our fibration a Clifford fibration and not a Hopf fibration, as our fibration is a geometrical construction. The simple algebraic properties of the fibration describe the geometrical properties of the kinematical algebras and groups as well as the spacetimes that are derived from them. We develop an algebraic framework that handles all possible kinematic algebras save one, the static algebra.
\end{abstract}

Key words: Clifford fibration; Hopf fibration; kinematic

2000 Mathematics Subject Classification: 11E88; 15A66; 53A17

As long as algebra and geometry have been separated, their progress have been slow and their uses limited; but when these two sciences have been united, they have lent each mutual forces, and have marched together towards perfection.

Joseph Louis Lagrange (1736-1813)

The nice role played by quaternions in describing rotations of Euclidean 3-dimensional space, and the beauty of the Hopf fibration of the 3-sphere, can be simply generalized for the study of $(1+1)$ kinematics. It is the purpose of this paper to show how this can be done. We can let $\mathbf{i}, \mathbf{j}$, and $\mathbf{k}$ denote the basis of the imaginary part of a generalized quaternion number system so that they also describe a basis for any given kinematical algebra save the static algebra. The space of unit quaternions (under a suitable choice of norm) then describes a " 3 -sphere". If $q$ is a point on this sphere, then the Hopf flows $\mathbf{i} q, \mathbf{j} q$, and $\mathbf{k} q$ describe fibrations of the sphere where the base spaces are the space of events, the space of space-like geodesics, or the space of time-like geodesics. The description given below is of a unified approach to all kinematical algebras (save the state algebra) as well as to the three classical Riemannian surfaces of constant curvature.

\section{Possible kinematics}

It is the purpose of this section to briefly review Bacry and Lévy-Leblond's work on possible kinematics. Bacry and Lévy-Leblond's investigations into the nature of all possible Lie algebras for kinematical groups given three basic principles

(i) space is isotropic and spacetime is homogeneous;

(ii) parity and time-reversal are automorphisms of the kinematical group;

(iii) the one-dimensional subgroups generated by the boosts are non-compact 
Table 1. The 11 possible kinematical groups.

\begin{tabular}{c|c}
\hline Symbol & Name \\
\hline$d S$ & de Sitter groups \\
$a d S$ & anti-de Sitter groups \\
$M$ & Minkowski groups \\
$M_{+}$ & expanding Minkowski groups \\
$M^{\prime}$ & para-Minkowski groups \\
$C$ & Carroll groups \\
$N_{+}$ & expanding Newtonian Universe groups \\
$N_{-}$ & oscillating Newtonian Universe groups \\
$G$ & Galilei group \\
$S d S$ & static de Sitter Universe groups \\
$S t$ & static Universe group
\end{tabular}

Table 2. The characteristic Lie brackets for the kinematical Lie algebras.

\begin{tabular}{c|c|c|c|c|c|c|c|c|c|c|c}
\hline \hline & $d S$ & $a d S$ & $M$ & $M_{+}$ & $M^{\prime}$ & $C$ & $N_{+}$ & $N_{-}$ & $G$ & $S d S$ & $S t$ \\
\hline$[H, P]$ & $-K$ & $K$ & 0 & $-K$ & $K$ & 0 & $-K$ & $K$ & 0 & $-K$ & 0 \\
{$[K, H]$} & $P$ & $P$ & $P$ & 0 & 0 & 0 & $P$ & $P$ & $P$ & 0 & 0 \\
{$[K, P]$} & $H$ & $H$ & $H$ & $H$ & $H$ & $H$ & 0 & 0 & 0 & 0 & 0 \\
\hline \hline
\end{tabular}

gave rise to 11 possible kinematical algebras. Restricting our attention to 2-dimensional spacetimes we still obtain the same 11 kinds of algebras (see [8]), where each of the kinematical groups is generated by its inertial transformations as well as its spacetime translations. These groups consist of the de Sitter groups and their contractions. The physical nature of a contracted group is determined by the nature of the contraction itself, along with the nature of the parent de Sitter group. The names of the 2-dimensional groups are given in Table 1.

In this paper we will restrict our attention to 2-dimensional spacetimes. So let $K$ denote the generator of the inertial transformations, $H$ the generator of time translations, and $P$ the generator of space translations. The kinematical algebras are determined by the structure constants $p, h$, and $k$ that are given by the commutators

$$
[K, H]=p P, \quad[K, P]=h H, \quad \text { and } \quad[H, P]=k K .
$$

If we normalize the structure constants to lie in the set $\{-1,0,1\}$, then the characteristic Lie brackets for the kinematical Lie algebras are as given in Table 2 (see [1]).

We will follow Herranz, Ortega and Santander (see [5]) and reduce the number of structure constants from three to two as follows. The kinematical algebras $d S$, adS, $M, N_{+}, N_{-}$, and $G$ (after rescaling) are determined by the structure constants $\kappa_{1}$ and $\kappa_{2}$ that are given by the commutators

$$
[K, H]=P, \quad[K, P]=-\kappa_{2} H, \quad \text { and } \quad[H, P]=\kappa_{1} K .
$$

The constant $\kappa_{1}= \pm \frac{1}{\tau^{2}}$ gives the spacetime curvature $\kappa_{1}$ as well as the universe (time) radius $\tau$, and the constant $\kappa_{2}=-\frac{1}{c^{2}}$ gives the speed of $\operatorname{light}^{1} c$. For the de Sitter groups $\kappa_{1}<0$ and $\kappa_{2}<0$, while for the anti-de Sitter groups $\kappa_{1}>0$ and $\kappa_{2}<0$. The remaining kinematical algebras (save for $S t)$ can be obtained by group contractions $\left(\kappa_{1} \rightarrow 0\right.$ or $\left.\kappa_{2} \rightarrow 0\right)$ in possible conjunction with the symmetries $S_{P}, S_{H}$, and $S_{K}$ :

$$
S_{P}:\{K \leftrightarrow H\}: \quad[K, H]=-P, \quad[K, P]=\kappa_{1} H, \quad \text { and } \quad[H, P]=-\kappa_{2} K,
$$

\footnotetext{
${ }^{1}$ See [4]. We will also demonstrate that $\kappa_{2}=-\frac{1}{c^{2}}$ and that $\kappa_{1}$ is the spacetime curvature later on in this paper.
} 


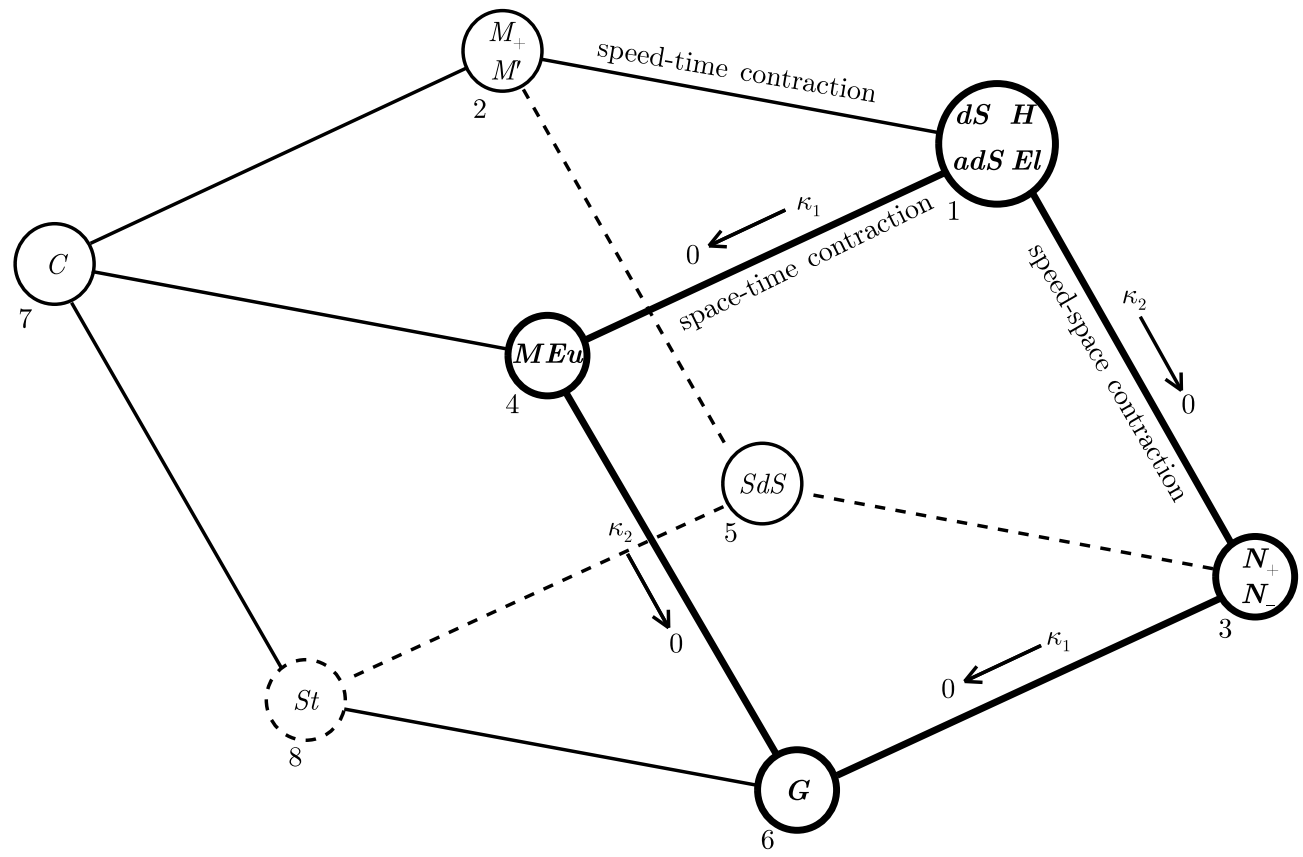

Figure 1. The 11 kinematical and 3 non-kinematical groups.

Table 3. The characteristic Lie brackets for the non-kinematical Lie algebras.

\begin{tabular}{c|c|c|c}
\hline \hline & $E l$ & $E u$ & $H$ \\
\hline$[H, P]$ & $K$ & 0 & $-K$ \\
{$[K, H]$} & $P$ & $P$ & $P$ \\
{$[K, P]$} & $-H$ & $-H$ & $-H$ \\
\hline \hline
\end{tabular}

$$
S_{H}:\{K \leftrightarrow P\}: \quad[K, H]=-\kappa_{1} P, \quad[K, P]=\kappa_{2} H, \quad \text { and } \quad[H, P]=-K
$$

and

$$
S_{K}:\{H \leftrightarrow P\}: \quad[K, H]=-\kappa_{2} P, \quad[K, P]=H, \quad \text { and } \quad[H, P]=-\kappa_{1} K
$$

See Fig. 1 for an illustration of how the different groups are related via contractions and symmetries: $E l, E u$, and $H$ denote the (non-kinematical) isometry groups of the elliptical, Euclidean, and hyperbolic planes (of constant curvature $\kappa_{1}$ ) respectively (see Table 3 ).

For example, if $\kappa_{2} \rightarrow 0$ then $d S$ and $H$ contract to $N_{+}$while adS and $E l$ contract to $N_{-}$, while if $\kappa_{1} \rightarrow 0$ then $d S$ and $a d S$ contract to $M$ while $H$ and $E l$ contract to $E u$. Similarly a space-time contraction sends either $M_{+}$or $M^{\prime}$ to $C$. In this paper we will specifically work with the nine kinds of groups indicated in bold in Fig. 1, since the other groups (save $S t$ ) are then easily obtained from these nine: the lie algebras of our nine groups have the commutators as given by (1). Henceforward we will not refer to the other algebras.

We can contract with respect to any subgroup, giving us three fundamental types of contraction: speed-space, speed-time, and space-time contractions, corresponding respectively to contracting to the subgroups generated by $H, P$, and $K$.

Speed-space contractions. We make the substitutions $K \rightarrow \epsilon K$ and $P \rightarrow \epsilon P$ into the Lie algebra and then calculate the singular limit of the Lie brackets as $\epsilon \rightarrow 0$. Physically the velocities are small when compared to the speed of light, and the spacelike intervals are small when compared to the timelike intervals. Geometrically we are describing spacetime near a timelike 
Table 4. The 3 basic symmetries are given as reflections of Fig. 1.

\begin{tabular}{c|c|l}
\hline Symmetry & Reflection across face & Corresponding group transformations \\
\hline \hline$S_{H}$ & 1378 & $M \longleftrightarrow M^{\prime}, E u \longleftrightarrow M_{+}, G \longleftrightarrow S d S$ \\
$S_{P}$ & 1268 & $C \longleftrightarrow S d S, M \longleftrightarrow N_{+}, E u \longleftrightarrow N_{-}$ \\
$S_{K}$ & 1458 & $C \longleftrightarrow M_{+} \longleftrightarrow N_{-}, M^{\prime} \longleftrightarrow N_{+}$ \\
\hline
\end{tabular}

Table 5. Important classes of kinematical groups and their geometrical configurations in Fig. 1.

\begin{tabular}{l|l}
\hline Class of groups & Face \\
\hline \hline Relative-time & 1247 \\
Absolute-time & 3568 \\
Relative-space & 1346 \\
Absolute-space & 2578 \\
Cosmological & 1235 \\
Local & 4678 \\
\hline
\end{tabular}

geodesic, as we are contracting to the subgroup that leaves this worldline invariant, and so are passing from relativistic to absolute time.

Speed-time contractions. We make the substitutions $K \rightarrow \epsilon K$ and $H \rightarrow \epsilon H$ into the Lie algebra and then calculate the singular limit of the Lie brackets as $\epsilon \rightarrow 0$. Physically the velocities are small when compared to the speed of light, and the timelike intervals are small when compared to the spacelike intervals. Geometrically we are describing spacetime near a spacelike geodesic, as we are contracting to the subgroup that leaves invariant this set of simultaneous events, and so are passing from relativistic to absolute space. Such a spacetime may be of limited physical interest, as we are only considering intervals connecting events that are not causally related.

Space-time contractions. We make the substitutions $P \rightarrow \epsilon P$ and $H \rightarrow \epsilon H$ into the Lie algebra and then calculate the singular limit of the Lie brackets as $\epsilon \rightarrow 0$. Physically the spacelike and timelike intervals are small, but the boosts are not restricted. Geometrically we are describing spacetime near an event, as we are contracting to the subgroup that leaves invariant only this one event, and so we call the corresponding kinematical group a local group as opposed to a cosmological group.

\section{Generalized complex numbers}

The generalized complex numbers are not new to physics or mathematics (see [12] for example). It is the purpose of this section to introduce these numbers to the reader who is not already familiar with them.

Definition 1. By the complex number plane $\mathbb{C}_{\kappa}$ we will mean the set of numbers of the form $\left\{z=x+i y \mid(x, y) \in \mathbb{R}^{2}\right.$ and $\left.i^{2}=-\kappa\right\}$, where the constant $\kappa$ is real and $i$ is not. $\mathbb{C}_{\kappa}$ is a real commutative algebra and also has zero divisors when $\kappa \leq 0$. The real part of $z$ is given by $\mathcal{R}(z)=x$ and the imaginary part by $\mathcal{I}(z)=y$.

Zero divisors play an important role in determining the conformal structure of spacetime, and although it does not make good algebraic sense to divide by them, one can form the Riemann sphere $\Sigma_{\kappa}$.

Definition 2. Let $\Sigma_{\kappa}$ denote the Riemann sphere consisting of the set of all equivalence classes $\left[\frac{A}{B}\right]$ of complex ratios $\frac{A}{B}$, where $A, B \in \mathbb{C}_{\kappa}$ and where either $A$ or $B$ is not a zero-divisor: $\frac{A}{B} \sim \frac{C}{D} \Longleftrightarrow A=\mu C$ and $B=\mu D$ for some $\mu \in \mathbb{C}_{\kappa}$ where $\mu$ is not a zero divisor. 


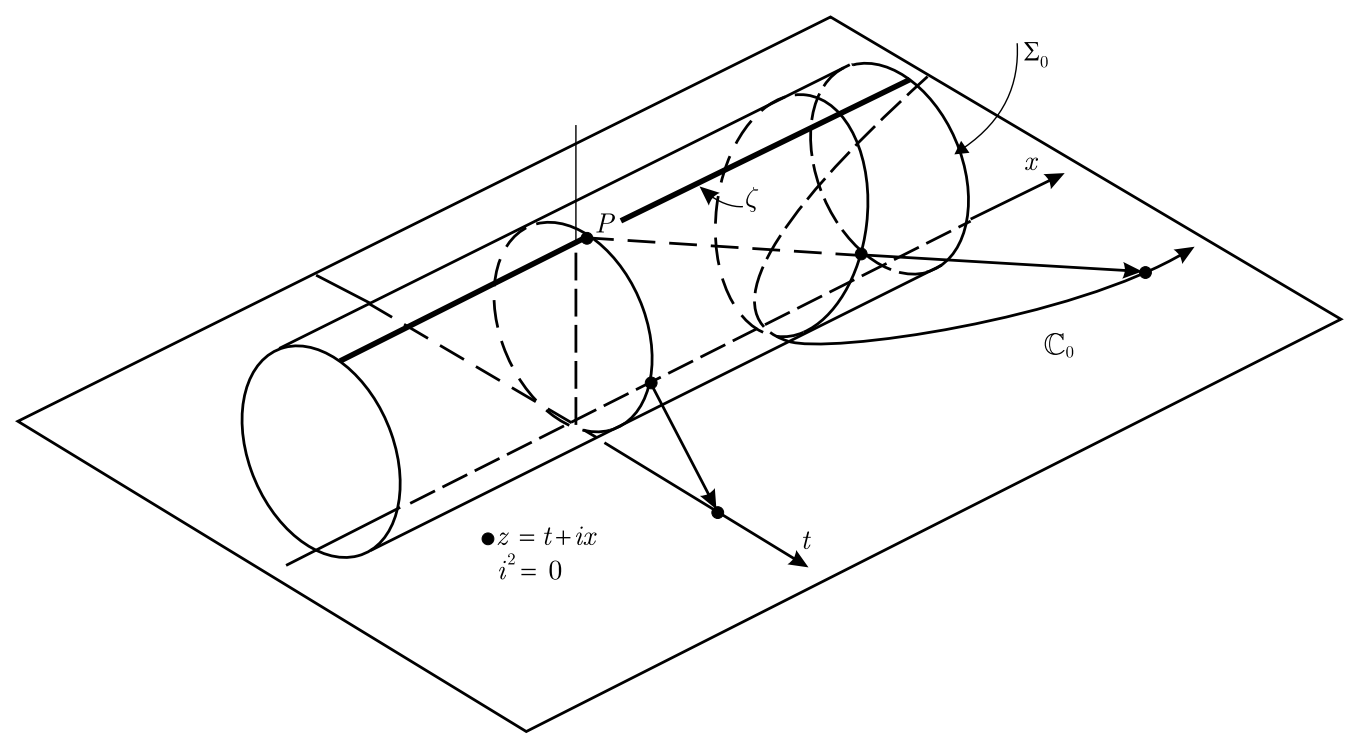

Figure 2. The Riemann sphere $\Sigma_{0}$.

We can describe $\Sigma_{\kappa}$ through stereographic projection, giving a circular cylinder when $\kappa=0$ and a hyperboloid of one sheet when $\kappa<0$ (see [12] for details). Fig. 2 shows such a construction for $\Sigma_{0}$ : Here we are projecting from the point $P$ onto the complex number plane $\mathbb{C}_{0}$ (where the zero divisors consists of all purely imaginary numbers), so that numbers of the form $\frac{1}{a i}$ correspond to the line $\zeta$ of "infinities" on $\Sigma_{0}$. So $P=\left[\frac{1}{0}\right]$, for example.

The unit circle $z \bar{z}=x^{2}+\kappa y^{2}=1$ in $\mathbb{C}_{\kappa}$, where $\bar{z}=x-i y$, is determined by the Hermitian metric $d z d \bar{z}=d x^{2}+\kappa d y^{2}$. The unit circle can be used to define the cosine

$$
C_{\kappa}(\phi)= \begin{cases}\cos (\sqrt{\kappa} \phi), & \text { if } \kappa>0, \\ 1, & \text { if } \kappa=0, \\ \cosh (\sqrt{-\kappa} \phi), & \text { if } \kappa<0\end{cases}
$$

and sine

$$
S_{\kappa}(\phi)= \begin{cases}\frac{1}{\sqrt{\kappa}} \sin (\sqrt{\kappa} \phi), & \text { if } \kappa>0, \\ \phi, & \text { if } \kappa=0, \\ \frac{1}{\sqrt{-\kappa}} \sinh (\sqrt{-\kappa} \phi), & \text { if } \kappa<0\end{cases}
$$

functions. Here $e^{i \phi}=C_{\kappa}(\phi)+i S_{\kappa}(\phi)$ is a point on the connected component of the unit circle containing 1 , and $\phi$ is the signed distance from 1 to $e^{i \phi}$ along the circular arc, defined modulo the length $\frac{2 \pi}{\sqrt{\kappa}}$ of the unit circle when $\kappa>0^{2}$. The power series for these analytic trigonometric functions are as follows:

$$
\begin{aligned}
& C_{\kappa}(\phi)=1-\frac{1}{2 !} \kappa \phi^{2}+\frac{1}{4 !} \kappa^{2} \phi^{4}+\cdots, \\
& S_{\kappa}(\phi)=\phi-\frac{1}{3 !} \kappa \phi^{3}+\frac{1}{5 !} \kappa^{2} \phi^{5}+\cdots .
\end{aligned}
$$

Note that $C_{\kappa}^{2}(\phi)+\kappa S_{\kappa}^{2}(\phi)=1$. We also have that

$$
\frac{d}{d \phi} C_{\kappa}(\phi)=-\kappa S_{\kappa}(\phi), \quad \frac{d}{d \phi} S_{\kappa}(\phi)=C_{\kappa}(\phi) .
$$

\footnotetext{
${ }^{2}$ When $\kappa=0$ the distance along the unit circle $x^{2}=1$ is defined by $d s^{2}=d y^{2}$, as the Hermitian metric $d s^{2}=d x^{2}+\kappa d y^{2}=d x^{2}$ vanishes on the unit circle. This is an instance where it is advantageous to rescale a metric.
} 
Definition 3. Let $U_{\kappa}(1)$ denote the group (under multiplication) of unit complex numbers in $\mathbb{C}_{\kappa}$.

Before we proceed it might be insightful to see how the algebraic structure of $\mathbb{C}_{\kappa_{2}}$ is useful in describing kinematics for the kinematical groups $M$ (flat Minkowski spacetimes) and $G$ (flat Galilean spacetime). We will demonstrate the well known fact that classical kinematics is a limiting case of relativistic kinematics. The Lorentz transformation

$$
\left(\begin{array}{l}
x^{\prime} \\
t^{\prime}
\end{array}\right)=\frac{1}{\sqrt{1-v^{2} / c^{2}}}\left(\begin{array}{cc}
1 & -v \\
-\frac{v}{c^{2}} & 1
\end{array}\right)\left(\begin{array}{l}
x \\
t
\end{array}\right)
$$

can be simply written in complex notation as $z^{\prime}=e^{i \theta} z$ in $C_{\kappa_{2}}$, where $z^{\prime}=t^{\prime}+i x^{\prime}, z=t+i x$, and $\kappa_{2}=-\frac{1}{c^{2}}$. This is because rotation about the origin through the angle $\theta$ in the complex plane $\mathbb{C}_{\kappa_{2}}$ can be written as the linear transformation (or boost)

$$
z \mapsto e^{i \theta} z \rightsquigarrow\left(\begin{array}{c}
x \\
t
\end{array}\right) \mapsto\left(\begin{array}{cc}
C_{\kappa_{2}}(\theta) & S_{\kappa_{2}}(\theta) \\
-\kappa_{2} S_{\kappa_{2}}(\theta) & C_{\kappa_{2}}(\theta)
\end{array}\right)\left(\begin{array}{l}
x \\
t
\end{array}\right)
$$

in $\mathbb{R}^{2}$, where $\theta=T_{\kappa_{2}}^{-1}(-v)$, so that

$$
\begin{aligned}
& T_{\kappa_{2}}(\theta)=\frac{S_{\kappa_{2}}(\theta)}{C_{\kappa_{2}}(\theta)}=-v \quad \text { and } \\
& C_{\kappa_{2}}^{2}(\theta)+\kappa_{2} S_{\kappa_{2}}^{2}(\theta)=C_{\kappa_{2}}^{2}(\theta)-\frac{1}{c^{2}} S_{\kappa_{2}}^{2}(\theta)=1 .
\end{aligned}
$$

On the other hand the Galilean transformation

$$
\left(\begin{array}{l}
x^{\prime} \\
y^{\prime}
\end{array}\right)=\left(\begin{array}{cc}
1 & -v \\
0 & 1
\end{array}\right)\left(\begin{array}{l}
x \\
t
\end{array}\right)
$$

can be simply written in complex notation as $z^{\prime}=e^{i \theta} z$ in $C_{0}$, where $\kappa_{2}=-\frac{1}{c^{2}}=0$ as the speed of light is infinite in Galilean spacetime. Note that $C_{0}(\theta)=1$ and $S_{0}(\theta)=\theta$, so that rotation about the origin through the angle $\theta$ can be written as the linear "shift" transformation (or boost)

$$
z \mapsto e^{i \theta} z \rightsquigarrow\left(\begin{array}{l}
x \\
t
\end{array}\right) \mapsto\left(\begin{array}{cc}
C_{0}(\theta) & S_{0}(\theta) \\
0 & C_{0}(\theta)
\end{array}\right)\left(\begin{array}{l}
x \\
t
\end{array}\right)=\left(\begin{array}{cc}
1 & -\theta \\
0 & 1
\end{array}\right)\left(\begin{array}{l}
x \\
t
\end{array}\right)
$$

in $\mathbb{R}^{2}$, where $\theta=-v$.

The speed-space contraction $\kappa_{2} \rightarrow 0$ (passing from relative- to absolute-time) takes Minkowski spacetime to Galilean spacetime, as $C_{\kappa_{2}}(\theta) \rightarrow 1$ and $S_{\kappa_{2}}(\theta) \rightarrow \theta$. The unit circle $z \bar{z}=1$ in Minkowski spacetime, a hyperbola, transforms into the "degenerate" hyperbola given by $t= \pm 1$. The light cone in Minkowski spacetime, given by $t= \pm \frac{1}{c} x$, transforms into the "light cone" $t=0$ in Galilean spacetime, where $c=\infty$. Locally all spacetimes are equivalent to Minkowski or Galilean spacetime via space-time contractions where $\kappa_{1} \rightarrow 0$ (passing from a cosmological to a local group) so we see that $\kappa_{2}=-\frac{1}{c^{2}}$.

Although we will not need the theorem stated below, the reader might be interested in seeing it. Undoubtedly this theorem was known to Yaglom.

Theorem 1 (Yaglom). Let $f(t, x)=u(t, x)+i v(t, x)$ and $i^{2}=-\kappa$, where the partial derivatives of $u$ and $v$ are continuous on an open set. Then $f$ is holomorphic on that open set if and only if the Cauchy-Riemann equations

$$
u_{t}=v_{x}, \quad u_{x}=-\kappa v_{t}
$$

are satisfied. Furthermore, $f$ is conformal at any point $w$ where $f^{\prime}(w)$ is not a zero-divisor ${ }^{3}$.

The usual proofs for $\kappa=1$ apply.

\footnotetext{
${ }^{3}$ On the complex plane $\mathbb{C}_{\kappa}$, the argument function is only defined on the set of non-zero divisors, for a non-zero divisor $w$ can be written $w=r e^{i \theta}$ where $r$ is the $\operatorname{norm} \operatorname{sgn}(w \bar{w}) \sqrt{|w \bar{w}|^{2}}$ of $w$ and $\operatorname{Arg}\left(r e^{i \theta}\right)=\theta$, see [3].
} 


\section{A very brief review of some work by Ballesteros, Herranz, Ortega and Santander}

It is the purpose of this section to introduce some material by Ballesteros, Herranz, Ortega and Santander that we will refer to in subsequent sections. A real matrix representation for a kinematical Lie algebra, denoted by $s o_{\kappa_{1}, \kappa_{2}}(3)$, is given by

$$
H=\left(\begin{array}{ccc}
0 & -\kappa_{1} & 0 \\
1 & 0 & 0 \\
0 & 0 & 0
\end{array}\right), \quad P=\left(\begin{array}{ccc}
0 & 0 & -\kappa_{1} \kappa_{2} \\
0 & 0 & 0 \\
1 & 0 & 0
\end{array}\right), \quad \text { and } \quad K=\left(\begin{array}{ccc}
0 & 0 & 0 \\
0 & 0 & -\kappa_{2} \\
0 & 1 & 0
\end{array}\right)
$$

where the structure constants are given by the commutators (1)

$$
[K, H]=P, \quad[K, P]=-\kappa_{2} H, \quad \text { and } \quad[H, P]=\kappa_{1} K .
$$

Elements of a corresponding kinematical Lie group, denoted by $S O_{\kappa_{1}, \kappa_{2}}(3)$, are given by reallinear, orientation-preserving isometries of $\left.\mathbb{R}^{3}=\{(y, t, x))\right\}$ imbued with the (possibly indefinite or degenerate) metric $d s^{2}=d y^{2}+\kappa_{1} d t^{2}+\kappa_{1} \kappa_{2} d x^{2}$. The one-parameter subgroups $\mathcal{H}, \mathcal{P}$, and $\mathcal{K}$ generated respectively by $H, P$, and $K$ consist of matrices of the form

$$
e^{\alpha H}=\left(\begin{array}{ccc}
C_{\kappa_{1}}(\alpha) & -\kappa_{1} S_{\kappa_{1}}(\alpha) & 0 \\
S_{\kappa_{1}}(\alpha) & C_{\kappa_{1}}(\alpha) & 0 \\
0 & 0 & 1
\end{array}\right), \quad e^{\beta P}=\left(\begin{array}{ccc}
C_{\kappa_{1} \kappa_{2}}(\beta) & 0 & -\kappa_{1} \kappa_{2} S_{\kappa_{1} \kappa_{2}}(\beta) \\
0 & 1 & 0 \\
S_{\kappa_{1} \kappa_{2}}(\beta) & 0 & C_{\kappa_{1} \kappa_{2}}(\beta)
\end{array}\right)
$$

and

$$
e^{\theta K}=\left(\begin{array}{ccc}
1 & 0 & 0 \\
0 & C_{\kappa_{2}}(\theta) & -\kappa_{2} S_{\kappa_{2}}(\theta) \\
0 & S_{\kappa_{2}}(\theta) & C_{\kappa_{2}}(\theta)
\end{array}\right)
$$

Ballesteros, Herranz, Ortega and Santander have constructed spacetimes as homogeneous spaces $^{4}$ by looking at real representations of their motion groups $S O_{\kappa_{1}, \kappa_{2}}(3)$. The spaces $S O_{\kappa_{1}, \kappa_{2}}(3) / \mathcal{K}, S O_{\kappa_{1}, \kappa_{2}}(3) / \mathcal{H}$, and $S O_{\kappa_{1}, \kappa_{2}}(3) / \mathcal{P}$ are homogeneous spaces for $S O_{\kappa_{1}, \kappa_{2}}(3)$. When $S O_{\kappa_{1}, \kappa_{2}}(3)$ is a kinematical group, then $S O_{\kappa_{1}, \kappa_{2}}(3) / \mathcal{K}$ can be identified with the manifold of space-time translations, $S O_{\kappa_{1}, \kappa_{2}}(3) / \mathcal{P}$ the manifold of time-like geodesics, and $S O_{\kappa_{1}, \kappa_{2}}(3) / \mathcal{H}$ the manifold of space-like geodesics.

\section{Generalized quaternions}

The goal of this section is to develop a simple algebraic description of the kinematical algebras, using what we already know about the generalized complex numbers. To that end, we begin by putting the Hermitian norm $d z d \bar{z}=d z_{1} d \overline{z_{1}}+\kappa_{1} d z_{2} d \overline{z_{2}}$ on $\mathbb{C}_{\kappa_{2}}^{2} \equiv C_{\kappa_{2}} \times C_{\kappa_{2}}$, where $z=\left(z_{1}, z_{2}\right)$ is an element of $\mathbb{C}_{\kappa_{2}}^{2}$. The construction below follows a natural course based on the double covering of $S O(3)$ by $S U(2)$ as part of the geometry of the standard quaternions.

The Hermitian inner product is obtained as follows. Let $z=\left(z_{1}, z_{2}\right)$ and $w=\left(w_{1}, w_{2}\right)$. Then

$$
\begin{aligned}
\langle z, w\rangle & =\frac{1}{2}\left(|z+w|^{2}-|z|^{2}-|w|^{2}\right) \\
& =\frac{1}{2}\left(\left(z_{1}+w_{1}\right)\left(\overline{z_{1}}+\overline{w_{1}}\right)+\kappa_{1}\left(z_{2}+w_{2}\right)\left(\overline{z_{2}}+\overline{w_{2}}\right)-z_{1} \overline{z_{1}}-\kappa_{1} z_{2} \overline{z_{2}}-w_{1} \overline{w_{1}}-\kappa_{1} w_{2} \overline{w_{2}}\right)
\end{aligned}
$$

\footnotetext{
${ }^{4}$ See $[2,5,6]$, and also [4], where a special case of the group law is investigated, leading to a plethora of trigonometric identities.
} 


$$
=\frac{1}{2}\left(z_{1} \overline{w_{1}}+\overline{z_{1}} w_{1}+\kappa_{1} z_{2} \overline{w_{2}}+\kappa_{1} \overline{z_{2}} w_{2}\right)=x_{1} x_{2}+\kappa_{2} y_{1} y_{2}+\kappa_{1} u_{1} u_{2}+\kappa_{1} \kappa_{2} v_{1} v_{2},
$$

where $z_{1}=x_{1}+i y_{1}, z_{2}=u_{1}+i v_{1}, w_{1}=x_{2}+i y_{2}$, and $w_{2}=u_{2}+i v_{2}$. So in $\mathbb{R}^{4}$ we can write the inner product as

$$
\left(\begin{array}{llll}
x_{1} & y_{1} & u_{1} & v_{1}
\end{array}\right)\left(\begin{array}{cccc}
1 & 0 & 0 & 0 \\
0 & \kappa_{2} & 0 & 0 \\
0 & 0 & \kappa_{1} & 0 \\
0 & 0 & 0 & \kappa_{1} \kappa_{2}
\end{array}\right)\left(\begin{array}{l}
x_{2} \\
y_{2} \\
u_{2} \\
v_{2}
\end{array}\right)=x_{1} x_{2}+\kappa_{2} y_{1} y_{2}+\kappa_{1} u_{1} u_{2}+\kappa_{1} \kappa_{2} v_{1} v_{2} .
$$

Definition 4. By the set of generalized quaternions $\mathbb{H}_{\kappa_{1}, \kappa_{2}}$ (or simply quaternions for short) we will mean the set of numbers of the form $\left\{(x+\mathbf{i} y+\mathbf{j} u+\mathbf{k} v) \mid \mathbf{i}^{2}=-\kappa_{2}, \mathbf{j}^{2}=-\kappa_{1}, \mathbf{k}^{2}=-\kappa_{1} \kappa_{2}\right\}$ with the following product rules ${ }^{5}$

$$
\begin{array}{ll}
\mathbf{i j}=\mathbf{k}, & \mathbf{j i}=-\mathbf{k}, \\
\mathbf{j} \mathbf{k}=\kappa_{1} \mathbf{i}, & \mathbf{k j}=-\kappa_{1} \mathbf{i}, \\
\mathbf{k} \mathbf{i}=\kappa_{2} \mathbf{j}, & \mathbf{i k}=-\kappa_{2} \mathbf{j} .
\end{array}
$$

We will show below that $\mathbb{H}_{\kappa_{1}, \kappa_{2}}$ is a real associative algebra over the reals and that the pure quaternions represent the kinematical algebras given by equation (1).

If $q=x+\mathbf{i} y+\mathbf{j} u+\mathbf{k} v$, then $q \bar{q}=x^{2}+\kappa_{2} y^{2}+\kappa_{1} u^{2}+\kappa_{1} \kappa_{2} v^{2}$, where $\bar{q}=x-\mathbf{i} y-\mathbf{j} u-\mathbf{k} v$. So if we identify points of $\mathbb{H}_{\kappa_{1}, \kappa_{2}}$ with points of $\mathbb{C}_{\kappa_{2}}^{2}=\left\{\left(z_{1}, z_{2}\right)\right\}$ by the correspondence

$$
x+\mathbf{i} y+\mathbf{j} u+\mathbf{k} v=z_{1}+z_{2} \mathbf{j} \rightsquigarrow\left(z_{1}, z_{2}\right),
$$

where $z_{1}=x+i y$ and $z_{2}=u+i v$ (in terms of quaternions we can think of $z_{1}$ and $z_{2}$ as $z_{1}=x+\mathbf{i} y$ and $\left.z_{2}=u+\mathbf{i} v\right)$, then the norm of $q$ corresponds to the norm of $\left(z_{1}, z_{2}\right)$.

Definition 5. Let $S U_{\kappa_{1}, \kappa_{2}}(2)$ denote the group of all matrices of the form

$$
\left(\begin{array}{cc}
z_{1} & z_{2} \\
-\kappa_{1} \overline{z_{2}} & \overline{z_{1}}
\end{array}\right)
$$

with determinant $z_{1} \overline{z_{1}}+\kappa_{1} z_{2} \overline{z_{2}}=1$. It was shown in [8] that $S U_{\kappa_{1}, \kappa_{2}}(2)$ is a double cover of $S O_{\kappa_{1}, \kappa_{2}}(3)$, and that $s u_{\kappa_{1}, \kappa_{2}}(2)$ consists of those elements $B$ of $M\left(2, \mathbb{C}_{\kappa_{2}}\right)$ such that $B^{\star} A+A B=0$ where $A$ is the matrix

$$
A=\left(\begin{array}{cc}
\kappa_{1} & 0 \\
0 & 1
\end{array}\right) \text {. }
$$

We will see below that $s u_{\kappa_{1}, \kappa_{2}}(2)$ can be identified with the space of pure quaternions, a real algebra, and that finally the space of pure quaternions is a kinematical algebra.

Under the correspondence

$$
x+\mathbf{i} y+\mathbf{j} u+\mathbf{k} v \rightsquigarrow\left(\begin{array}{cc}
z_{1} & z_{2} \\
-\kappa_{1} \overline{z_{2}} & \overline{z_{1}}
\end{array}\right)
$$

the set of unit quaternions is identified with $S U_{\kappa_{1}, \kappa_{2}}(2)$. The context should make it clear as to whether elements of $S U_{\kappa_{1}, \kappa_{2}}(2)$ are to be treated as elements of $M\left(2, \mathbb{C}_{\kappa_{2}}\right)$ or as unit quaternions in $\mathbb{H}_{\kappa_{1}, \kappa_{2}}$. The inner product on $\mathbb{C}_{\kappa_{2}}^{2}$ corresponds in $\mathbb{H}_{\kappa_{1}, \kappa_{2}}$ to

$$
\left\langle q_{1}, q_{2}\right\rangle=\frac{1}{2}\left(q_{1} \overline{q_{2}}+q_{2} \overline{q_{1}}\right)=\frac{1}{2}\left(\left(z_{1}+z_{2} \mathbf{j}\right)\left(\overline{w_{1}}-w_{2} \mathbf{j}\right)+\left(w_{1}+w_{2} \mathbf{j}\right)\left(\overline{z_{1}}-z_{2} \mathbf{j}\right)\right)
$$

\footnotetext{
${ }^{5}$ See [11] for another description of the generalized quaternions.
} 


$$
=\frac{1}{2}\left(z_{1} \overline{w_{1}}+\overline{z_{1}} w_{1}+\kappa_{1} z_{2} \overline{w_{2}}+\kappa_{1} \overline{z_{2}} w_{2}\right)=\frac{1}{2}\left(|z+w|^{2}-|z|^{2}-|w|^{2}\right)=\langle z, w\rangle,
$$

since $\mathbf{j}(x+\mathbf{i} y)=(x-\mathbf{i} y) \mathbf{j}$ and $\mathbf{j}^{2}=-\kappa_{1}$.

We can see that $\mathbb{H}_{\kappa_{1}, \kappa_{2}}$ and the subspace of $M\left(2, \mathbb{C}_{\kappa_{2}}\right)$ consisting of all matrices of the form $\left(\begin{array}{cc}z_{1} & z_{2} \\ -\kappa_{1} \overline{z_{2}} & \overline{z_{1}}\end{array}\right)$ are isomorphic as algebras, for if $q_{1}=z_{1}+z_{2} \mathbf{j}$ and $q_{2}=w_{1}+w_{2} \mathbf{j}$ are two quaternions with corresponding matrices

$$
\left(\begin{array}{cc}
z_{1} & z_{2} \\
-\kappa_{1} \overline{z_{2}} & \overline{z_{1}}
\end{array}\right) \quad \text { and } \quad\left(\begin{array}{cc}
w_{1} & w_{2} \\
-\kappa_{1} \overline{w_{2}} & \overline{w_{1}}
\end{array}\right)
$$

then

$$
q_{1}+q_{2} \rightsquigarrow\left(\begin{array}{cc}
z_{1} & z_{2} \\
-\kappa_{1} \overline{z_{2}} & \overline{z_{1}}
\end{array}\right)+\left(\begin{array}{cc}
w_{1} & w_{2} \\
-\kappa_{1} \overline{w_{2}} & \overline{w_{1}}
\end{array}\right)
$$

and

$$
q_{1} q_{2} \rightsquigarrow\left(\begin{array}{cc}
z_{1} & z_{2} \\
-\kappa_{1} \overline{z_{2}} & \overline{z_{1}}
\end{array}\right)\left(\begin{array}{cc}
w_{1} & w_{2} \\
-\kappa_{1} \overline{w_{2}} & \overline{w_{1}}
\end{array}\right)=\left(\begin{array}{cc}
z_{1} w_{1}-\kappa_{1} z_{2} \overline{w_{2}} & z_{1} w_{2}+z_{2} \overline{w_{1}} \\
-\kappa_{1} \overline{z_{2}} w_{1}-\kappa_{1} \overline{z_{1} w_{2}} & \overline{z_{1} w_{1}}-\kappa_{1} \overline{z_{2}} w_{2}
\end{array}\right),
$$

since

$$
\left(z_{1}+z_{2} \mathbf{j}\right)\left(w_{1}+w_{2} \mathbf{j}\right)=\left(z_{1} w_{1}-\kappa_{1} z_{2} \overline{w_{2}}\right)+\left(z_{1} w_{2}+z_{2} \overline{w_{1}}\right) \mathbf{j}
$$

Definition 6. We define the unit one-sphere and two-sphere ${ }^{6}$

$$
\begin{aligned}
& S_{\kappa_{2}}^{1}=U_{\kappa_{2}}(1)=\left\{z \in \mathbb{C}_{\kappa_{2}},|z|=1\right\} \rightsquigarrow\left\{e^{\mathbf{i} \theta}\right\} \subset \mathbb{H}_{\kappa_{1}, \kappa_{2}}, \\
& S_{\kappa_{1}, \kappa_{2}}^{3}=\left\{(z, w) \in \mathbb{C}_{\kappa_{2}}^{2},|(z, w)|=1\right\} \rightsquigarrow\left\{q \in \mathbb{H}_{\kappa_{1}, \kappa_{2}}|| q \mid=1\right\},
\end{aligned}
$$

where the set of unit quaternions is given by numbers of the form $e^{\mathbf{i} y+\mathbf{j} u+\mathbf{k} v}$. So $S_{\kappa_{1}, \kappa_{2}}^{3}$ can be identified with $S U_{\kappa_{1}, \kappa_{2}}(2)$.

The plane spanned by 1 and $\mathbf{i}$ can be easily identified with $\mathbb{C}_{\kappa_{2}}$, and the intersection of this plane with the sphere of unit quaternions then corresponds to the unit circle of $\mathbb{C}_{\kappa_{2}}$. Similar remarks hold for $\mathbb{C}_{\kappa_{1}}$ or $\mathbb{C}_{\kappa_{1} \kappa_{2}}$ for the planes spanned by 1 and $\mathbf{j}$ or 1 and $\mathbf{k}$ respectively. So $e^{\mathbf{i} t}$, $e^{\mathbf{j} t}$, and $e^{\mathrm{k} t}$ are all unit quaternions.

If $a$ is a unit quaternion, then $a^{-1}=\bar{a}$. Since $\overline{a b}=\bar{b} \bar{a}$ for any two quaternions $a$ and $b$, it follows that $\left|a q b^{-1}\right|=|q|$ for any quaternion $q$, provided that both $a$ and $b$ are unit quaternions. In fact, the generalized quaternions are a composition algebra, so that $\left|q_{1} q_{2}\right|=\left|q_{1}\right|\left|q_{2}\right|$. Also, $a \mathbf{q} a^{-1}$ is a pure quaternion since $\overline{a \mathbf{q} a^{-1}}=-a \mathbf{q} a^{-1}$, where $\mathbf{q}$ denotes the pure part of $q$. So the linear transformations (in terms of real coordinates)

$$
\mathbb{R}^{4} \rightarrow \mathbb{R}^{4} \text { defined by the automorphism } q \rightarrow a q b^{-1}
$$

and

$$
\mathbb{R}^{3} \rightarrow \mathbb{R}^{3} \text { defined by the inner automorphism } \mathbf{q} \rightarrow a \mathbf{q} a^{-1}
$$

respectively give rotations of $\mathbb{H}_{\kappa_{1}, \kappa_{2}}$ and the subspace of pure quaternions. It might appear then that $S U_{\kappa_{1}, \kappa_{2}}(2) \times S U_{\kappa_{1}, \kappa_{2}}(2)$ is a double cover of the group of rotations of $\mathbb{C}_{\kappa_{2}}^{2}$ with Hermitian metric $d z_{1} d \overline{z_{1}}+\kappa_{1} d z_{2} d \overline{z_{2}}$, as $(a, b)$ and $(-a,-b)$ represent the same rotation, but not all rotations

\footnotetext{
${ }^{6}$ The context should make it clear as to whether these spheres are to thought of in terms of generalized complex or quaternion numbers.
} 
can be so represented by such an automorphism. For example, if both $\kappa_{1}$ and $\kappa_{2}$ vanish, then rotations of $\mathbb{R}^{4}$ are of the form

$$
\left(\begin{array}{cccc}
1 & 0 & 0 & 0 \\
0 & m_{22} & m_{23} & m_{24} \\
0 & m_{32} & m_{33} & m_{34} \\
0 & m_{42} & m_{43} & m_{44}
\end{array}\right),
$$

and so the rotation group is 9-dimensional. Yet $S U_{\kappa_{1}, \kappa_{2}}(2) \times S U_{\kappa_{1}, \kappa_{2}}(2)$ has dimension 6 . Similarly $S U_{\kappa_{1}, \kappa_{2}}(2)$ is not a double cover for the rotation group for the subspace of pure quaternions ${ }^{7}$.

Let $s u_{\kappa_{1}, \kappa_{2}}(2)$ denote the Lie algebra of $S U_{\kappa_{1}, \kappa_{2}}(2)$. If we identify $S U_{\kappa_{1}, \kappa_{2}}(2)$ with $S_{\kappa_{1}, \kappa_{2}}^{3}$, the space of unit quaternions, then $s u_{\kappa_{1}, \kappa_{2}}(2)$ can be represented by the space of pure quaternions: For if $\mathbf{q}$ is a pure quaternion, then $e^{\mathbf{q}}$ is a unit quaternion, as $\mathbf{q} \overline{\mathbf{q}}=\overline{\mathbf{q}} \mathbf{q}$ so that $e^{\mathbf{q}} \overline{e^{\mathbf{q}}}=e^{\mathbf{q}} e^{\overline{\mathbf{q}}}=$ $e^{\mathbf{q}+\overline{\mathbf{q}}}=e^{\mathbf{0}}=1 . S U_{\kappa_{1}, \kappa_{2}}(2)$ acts on its Lie algebra $s u_{\kappa_{1}, \kappa_{2}}(2)$ by the inner automorphism $\mathbf{p} \mapsto e^{\frac{\theta}{2} \mathbf{q}} \mathbf{p} e^{-\frac{\theta}{2} \mathbf{q}}$ where both $\mathbf{p}$ and $\mathbf{q}$ are pure quaternions. Since

$$
\left.\frac{d}{d \theta}\right|_{\theta=0} e^{\frac{\theta}{2} \mathbf{q}} \mathbf{p} e^{-\frac{\theta}{2} \mathbf{q}}=\frac{1}{2}(\mathbf{q} \mathbf{p}-\mathbf{p q})=\frac{1}{2}[\mathbf{q}, \mathbf{p}],
$$

then

$$
\begin{aligned}
& \frac{1}{2}[\mathbf{i}, \mathbf{j}]=\left.\frac{d}{d \theta}\right|_{\theta=0} e^{\frac{\theta}{2} \mathbf{i}} \mathbf{j} e^{-\frac{\theta}{2} \mathbf{i}}=\left.\frac{d}{d \theta}\right|_{\theta=0} e^{\frac{\theta}{2} \mathbf{i}} e^{\frac{\theta}{2} \mathbf{i}} \mathbf{j}=\mathbf{i} \mathbf{j}=\mathbf{k}, \\
& \frac{1}{2}[\mathbf{i}, \mathbf{k}]=\left.\frac{d}{d \theta}\right|_{\theta=0} e^{\frac{\theta}{2} \mathbf{i}} \mathbf{k} e^{-\frac{\theta}{2} \mathbf{i}}=\left.\frac{d}{d \theta}\right|_{\theta=0} e^{\frac{\theta}{2} \mathbf{i}} e^{\frac{\theta}{2} \mathbf{i}} \mathbf{k}=\mathbf{i k}=-\kappa_{2} \mathbf{j}, \\
& \frac{1}{2}[\mathbf{j}, \mathbf{k}]=\left.\frac{d}{d \theta}\right|_{\theta=0} e^{\frac{\theta}{2} \mathbf{j}} \mathbf{k} e^{-\frac{\theta}{2} \mathbf{j}}=\left.\frac{d}{d \theta}\right|_{\theta=0} e^{\frac{\theta}{2} \mathbf{j}} e^{\frac{\theta}{2} \mathbf{j}} \mathbf{k}=\mathbf{j} \mathbf{k}=\kappa_{1} \mathbf{i}
\end{aligned}
$$

as $\mathbf{j} z=\bar{z} \mathbf{j}$ and $\mathbf{k} z=\bar{z} \mathbf{k}$. We can then represent a given kinematical Lie algebra by

$$
K \rightsquigarrow 2 \mathbf{i}, \quad H \rightsquigarrow 2 \mathbf{j}, \quad \text { and } \quad P \rightsquigarrow 2 \mathbf{k} .
$$

In terms of the ordered basis $\left\{E_{1}, E_{2}, E_{3}\right\}=\{2 \mathbf{i}, 2 \mathbf{j}, 2 \mathbf{k}\}$ for $s u_{\kappa_{1}, \kappa_{2}}(2)$, the structure constants are given by $\left[E_{i}, E_{j}\right]=C_{i j}^{k} E_{k}$, and so the Killing form on $s u_{\kappa_{1}, \kappa_{2}}(2)$ is given by $g_{i j}=$ $\sum_{r, s} C_{i s}^{r} C_{j r}^{s}$ or

$$
\left(g_{i j}\right)=-2\left(\begin{array}{ccc}
\kappa_{2} & 0 & 0 \\
0 & \kappa_{1} & 0 \\
0 & 0 & \kappa_{1} \kappa_{2}
\end{array}\right) .
$$

The Killing form is preserved by the inner automorphism.

We may form three natural homogeneous spaces:

$$
\begin{aligned}
& S U_{\kappa_{1}, \kappa_{2}}(2) /\langle\mathbf{i}\rangle=S U_{\kappa_{1}, \kappa_{2}}(2) / \mathcal{K} \rightsquigarrow S_{\kappa_{1}, \kappa_{2}}^{3} / S_{\kappa_{2}}^{1}, \\
& S U_{\kappa_{1}, \kappa_{2}}(2) /\langle\mathbf{j}\rangle=S U_{\kappa_{1}, \kappa_{2}}(2) / \mathcal{H} \rightsquigarrow S_{\kappa_{1}, \kappa_{2}}^{3} / S_{\kappa_{1}}^{1}, \\
& S U_{\kappa_{1}, \kappa_{2}}(2) /\langle\mathbf{k}\rangle=S U_{\kappa_{1}, \kappa_{2}}(2) / \mathcal{P} \rightsquigarrow S_{\kappa_{1}, \kappa_{2}}^{3} / S_{\kappa_{1} \kappa_{2}}^{1} .
\end{aligned}
$$

The Killing form naturally determines a metric for each of these homogeneous spaces with respective inner products

$$
\left(\begin{array}{cc}
\kappa_{1} & 0 \\
0 & \kappa_{1} \kappa_{2}
\end{array}\right), \quad\left(\begin{array}{cc}
\kappa_{2} & 0 \\
0 & \kappa_{1} \kappa_{2}
\end{array}\right) \quad \text { and } \quad\left(\begin{array}{cc}
\kappa_{2} & 0 \\
0 & \kappa_{1}
\end{array}\right)
$$

\footnotetext{
${ }^{7}$ Note that $\mathbf{q} \overline{\mathbf{q}}=\kappa_{2} y^{2}+\kappa_{1} u^{2}+\kappa_{1} \kappa_{2} w^{2}$ so that, when both $\kappa_{1}$ and $\kappa_{2}$ vanish, the dimension of the rotation group of the pure quaternions is 9-dimensional, as any orientation preserving linear map of $\mathbb{R}^{3}$ is a rotation.
} 


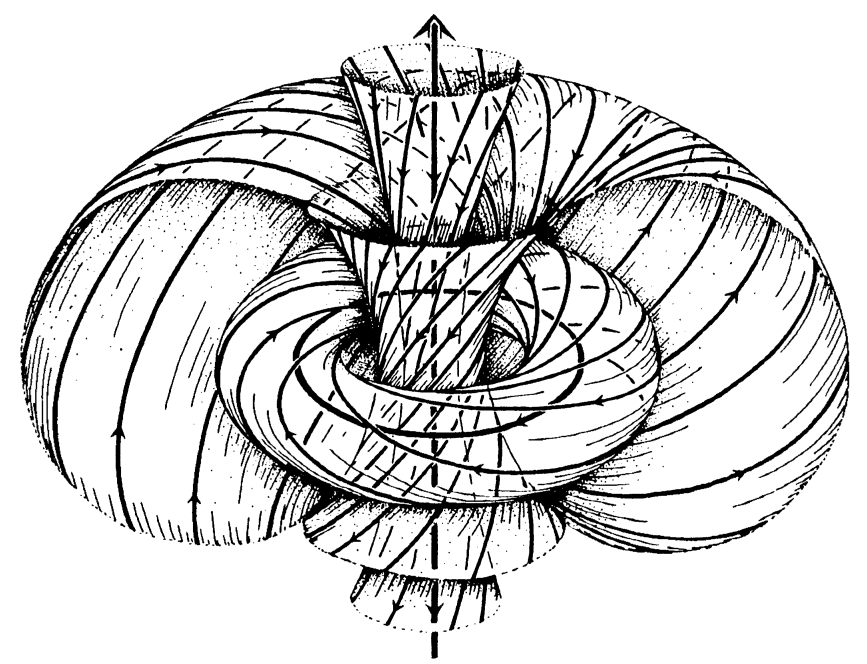

Figure 3. Stereographic projection of the Clifford fibration for $S^{3}$ onto $\mathbb{R}^{3}$. Image courtesy of [Penrose R., Rindler W., Spinors and space-time, Vol. 2, Spinor and twistor methods in space-time geometry, Cambridge University Press, 1986].

Following Ballesteros, Herranz, Ortega, and Santander we will rescale (even if $\kappa_{1}$ or $\kappa_{2}$ is equal to zero) so that, in fact, the respective inner products are as follows:

$$
\left(\begin{array}{cc}
1 & 0 \\
0 & \kappa_{2}
\end{array}\right), \quad\left(\begin{array}{cc}
1 & 0 \\
0 & \kappa_{1}
\end{array}\right) \quad \text { and } \quad\left(\begin{array}{cc}
\kappa_{2} & 0 \\
0 & \kappa_{1}
\end{array}\right)
$$

The resulting metrics can be indefinite as well as degenerate.

Theorem 2. Let $H, P$, and $K$ denote the respective generators for time translations, space translations, and boosts of the kinematical algebra with commutators

$$
[K, H]=P, \quad[K, P]=-\kappa_{2} H, \quad \text { and } \quad[H, P]=\kappa_{1} K .
$$

Then the kinematical algebra can be represented as the space of pure quaternions in $\mathbb{H}_{\kappa_{1}, \kappa_{2}}$ by

$$
K \rightsquigarrow 2 \mathbf{i}, \quad H \rightsquigarrow 2 \mathbf{j}, \quad \text { and } \quad P \rightsquigarrow 2 \mathbf{k} .
$$

If $S U_{\kappa_{1}, \kappa_{2}}(2)$ denotes the group of unit quaternions with lie algebra $s u_{\kappa_{1}, \kappa_{2}}(2)$, then $s u_{\kappa_{1}, \kappa_{2}}(2)$ is the space of pure quaternions and the homogeneous space $S U_{\kappa_{1}, \kappa_{2}} /\langle\mathbf{i}\rangle$ is the space of events, $S U_{\kappa_{1}, \kappa_{2}} /\langle\mathbf{j}\rangle$ is the space of space-like geodesics, and $S U_{\kappa_{1}, \kappa_{2}} /\langle\mathbf{k}\rangle$ is the space of time-like geodesics.

\section{The generalized Clifford fibration}

As pointed out by Urbantke (see [10]), Penrose has called the Clifford fibration an "element of the architecture of our world". This fibration can be used to describe two-level quantum systems, the harmonic oscillator, Taub-NUT space, Robinson congruences, helicity representations, magnetic monopoles, and the Dirac equation. By generalizing the Clifford fibration we will give yet another physical application by modeling all kinematical algebras save for the static algebra.

$S_{\kappa_{2}}^{1}$ acts freely and smoothly on $S_{\kappa_{1}, \kappa_{2}}^{3}$ by left multiplication: $q=z+w \mathbf{j} \mapsto e^{\mathbf{i} \theta} z+e^{\mathbf{i} \theta} w \mathbf{j}$. If $e^{\mathbf{i} \theta} q=q$, then $e^{\mathbf{i} \theta}=1$ since $|q|=1$ and so $q$ is not a zero-divisor. So $S_{\kappa_{1}, \kappa_{2}}^{3}$ is the total space of a principal $S_{\kappa_{2}}^{1}$ bundle. So what can we say about the base space of this bundle? 
We define $\mathbb{C}_{\kappa_{2}} \mathbb{P}_{1}$ as the space of all complex one-dimensional subspaces of the vector space $\mathbb{C}_{\kappa_{2}}^{2}$. Each subspace is uniquely described as the solution space to the complex linear equation $A z_{1}+B z_{2}=0$ where $\left[\frac{A}{B}\right]$ defines a point on the Riemann sphere $\Sigma_{\kappa_{2}}$. Note that $A$ and $B$ cannot both be zero-divisors, for then $\left[\frac{A}{B}\right]$ is not defined, and the set of points $\left(z_{1}, z_{2}\right)$ satisfying $A z_{1}+B z_{2}=0$ is no longer one-dimensional.

Alternatively, if we think of the complex line through the point $\left(z_{1}, z_{2}\right)$ as being given by points of the form $\lambda\left(z_{1}, z_{2}\right)$, where $\lambda$ takes all values in $\mathbb{C}_{\kappa_{2}}$, then we get a line precisely when either $z_{1}$ or $z_{2}$ is not a zero-divisor. For such a line we may let $A=z_{2} / z_{1}$ and $B=-1$ if $z_{1}$ is not a zero-divisor, and $A=-1$ and $B=z_{1} / z_{2}$ otherwise. Taking values in the Riemann sphere (so that "infinities" are allowed) we may always write $\left[\frac{A}{B}\right]=-\left[\frac{z_{2}}{z_{1}}\right]$. Note that distinct lines always intersect at the origin, but they may also intersect at points where both coordinates are zero-divisors, for $\lambda$ is allowed be a zero-divisor: So two points do not necessarily determine a unique line.

So we may identify $\mathbb{C}_{\kappa_{2}} \mathbb{P}_{1}$ with the Riemann sphere. A complex line will intersect $S_{\kappa_{1}, \kappa_{2}}^{3}$ exactly when $\left|z_{1}\right|^{2}+\kappa_{1}\left|z_{2}\right|^{2}=1$ for some point $\left(z_{1}, z_{2}\right)$ on the line: Let us call a line null if $\left|z_{1}\right|^{2}+\kappa_{1}\left|z_{2}\right|^{2}=0$ for all points $\left(z_{1}, z_{2}\right)$ on that line. So only the null lines do not intersect the unit sphere. A complex line that does intersect the unit sphere does so at points of the form $\left\{\left(e^{i \theta} z_{1}, e^{i \theta} z_{2}\right)\right\}$, where $\left(z_{1}, z_{2}\right)$ is any point belonging to the intersection. Let us denote by $\Sigma_{\kappa_{1}, \kappa_{2}}$ the subset of $\Sigma_{\kappa_{2}}$ corresponding to non-null lines. We will see below that $\Sigma_{\kappa_{1}, \kappa_{2}}$ is the homogeneous space $S_{\kappa_{1}, \kappa_{2}}^{3} / S_{\kappa_{2}}^{1}$.

So given a null line we must have that both $A z_{1}+B z_{2}=0$ and $\left|z_{1}\right|^{2}+\kappa_{1}\left|z_{2}\right|^{2}=0$ for all points $\left(z_{1}, z_{2}\right)$ on the line. Recall that $w \equiv\left[\frac{A}{B}\right]=-\left[\frac{z_{2}}{z_{1}}\right]$ represents a point on the Riemann sphere $\Sigma_{k}$. When $\kappa_{1}=0, \Sigma_{0, \kappa_{2}}$ is $\Sigma_{\kappa_{2}}$ with all infinities removed. If $\kappa_{1} \neq 0$, then null lines can exist only when $\kappa_{1}<0$, and in this case $\Sigma_{\kappa_{1}, \kappa_{2}}$ is $\Sigma_{\kappa_{2}}$ with all points of the form $|w|^{2}=-\frac{1}{\kappa_{1}}$ removed. It might be useful at this point to consider a familiar case: When $\kappa_{2}=1$ and $\kappa_{1}=1$, 0 , or -1 we have elliptic, euclidian, and hyperbolic geometry respectively. For elliptic geometry $\Sigma_{1,1}=\Sigma_{1}$ is the well known Riemann sphere. For the euclidean plane $\Sigma_{0,1}=\Sigma_{1} \backslash\{\infty\}$ is topologically a plane. And for the hyperbolic plane $\Sigma_{-1,1}=\Sigma_{1} \backslash S^{1}$ is topologically a union of two planes (each plane giving rise to a model of the hyperbolic plane).

We observe that the vectors $\mathbf{i} q, \mathbf{j} q$, and $\mathbf{k} q$ span the tangent space $T_{q}\left(S_{\kappa_{1}, \kappa_{2}}^{3}\right)$ of $S_{\kappa_{1}, \kappa_{2}}^{3}$ at $q$. For if $q \in S_{\kappa_{1}, \kappa_{2}}^{3}$, then $e^{\mathbf{i} t} q, e^{\mathbf{j} t} q$, and $e^{\mathbf{k} t} q$ are unit quaternions. Keeping $q$ fixed and letting $t$ vary, the respective tangents to the curves $e^{\mathbf{i} t} q, e^{\mathbf{j} t} q$, and $e^{\mathbf{k} t} q$ passing through $q$ are given by $\left.\frac{d}{d t}\right|_{t=0} e^{\mathbf{i} t} q=\mathbf{i} q,\left.\frac{d}{d t}\right|_{t=0} e^{\mathbf{j} t} q=\mathbf{j} q$, and $\left.\frac{d}{d t}\right|_{t=0} e^{\mathbf{k} t} q=\mathbf{k} q$. Now

$$
\begin{aligned}
& \mathbf{i} q=\mathbf{i}(x+\mathbf{i} y+\mathbf{j} u+\mathbf{k} v)=\mathbf{i} x-\kappa_{2} y+\mathbf{k} u-\kappa_{2} \mathbf{j} v=z_{1} \mathbf{i}+z_{2} \mathbf{k}, \\
& \mathbf{j} q=\mathbf{j}(x+\mathbf{i} y+\mathbf{j} u+\mathbf{k} v)=\mathbf{j} x-\mathbf{k} y-\kappa_{1} u+\kappa_{1} \mathbf{i} v=-\kappa_{1} \overline{z_{2}}+\overline{z_{1}} \mathbf{j}, \\
& \mathbf{k} q=\mathbf{k}(x+\mathbf{i} y+\mathbf{j} u+\mathbf{k} v)=\mathbf{k} x+\mathbf{j} \kappa_{2} y-\mathbf{i} \kappa_{1} u-\kappa_{1} \kappa_{2} v=-\kappa_{1} \overline{z_{2}} \mathbf{i}+\overline{z_{1}} \mathbf{k},
\end{aligned}
$$

and so $\mathbf{i} q, \mathbf{j} q$, and $\mathbf{k} q$ are linearly independent since $|q|=1$. Note that $\mathbf{i} q, \mathbf{j} q$ and $\mathbf{k} q$ are mutually orthogonal since

$$
\begin{aligned}
& \langle\mathbf{i} q, \mathbf{j} q\rangle=\mathbf{i} q(-\bar{q} \mathbf{j})+\mathbf{j} q(-\bar{q} \mathbf{i})=-\mathbf{i j}-\mathbf{j} \mathbf{i}=0 \\
& \langle\mathbf{i} q, \mathbf{k} q\rangle=\mathbf{i} q(-\bar{q} \mathbf{k})+\mathbf{k} q(-\bar{q} \mathbf{i})=-\mathbf{i} \mathbf{k}-\mathbf{k i}=0 \\
& \langle\mathbf{j} q, \mathbf{k} q\rangle=\mathbf{j} q(-\bar{q} \mathbf{k})+\mathbf{k} q(-\bar{q} \mathbf{j})=-\mathbf{j} \mathbf{k}-\mathbf{k j}=0
\end{aligned}
$$

However, the frame $\{\mathbf{i} q, \mathbf{j} q, \mathbf{k} q\}$ is not orthonormal since $|\mathbf{i} q|^{2}=\kappa_{2},|\mathbf{j} q|^{2}=\kappa_{1}$, and $|\mathbf{k} q|^{2}=\kappa_{1} \kappa_{2}$ (compare with the Killing form on $s u_{\kappa_{1}, \kappa_{2}}(2)$ ). The tangent plane spanned by $\mathbf{j} q$ and $\mathbf{k} q$ has the complex structure $\mathbb{C}_{\kappa_{2}}$ since multiplying $\mathbf{j} q$ on the left by $\mathbf{i}$ yields $\mathbf{k} q$. We also see that $S_{\kappa_{1}, \kappa_{2}}^{3}$ is parallelizable: This is no surprise however, since $S_{\kappa_{1}, \kappa_{2}}^{3}$ is topologically either $S^{3}, \mathbb{R}^{3}$, or $S^{1} \times \mathbb{R}^{2}$. 
Definition 7. The Clifford fibration is given by

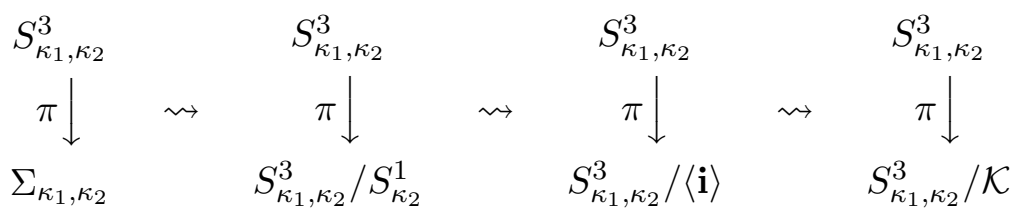

where $\pi^{-1}\left(\left[\frac{z_{1}}{z_{2}}\right]\right)=\left\{e^{\mathbf{i} \phi}\left(z_{1}+z_{2} \mathbf{j}\right)\right\}$.

This is a principal fiber bundle over $\Sigma_{\kappa_{1}, \kappa_{2}}$ with fiber given by $S_{\kappa_{2}}^{1}$ (the curve $e^{\mathbf{i} t} q$ is the fiber passing through $q$ ). The Clifford flow is given by the vector field $\chi_{\mathbf{i}}(q)=\mathbf{i} q$, and the canonical connection is determined by the horizontal planes spanned by $\mathbf{j} q$ and $\mathbf{k} q$ at each unit quaternion $q \in S_{\kappa_{1}, \kappa_{2}}^{3}$. Each such plane has the complex structure of $\mathbb{C}_{\kappa_{2}}$. Here $\Sigma_{\kappa_{1}, \kappa_{2}}$ is the spacetime for the kinematical algebra.

Similarly, we may form the fibrations

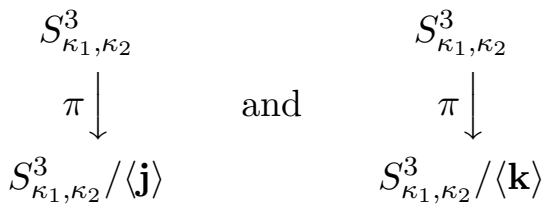

with respective Clifford flows given by $\chi_{\mathbf{j}}(q)=\mathbf{j} q$ and $\chi_{\mathbf{k}}(q)=\mathbf{k} q$. These fibrations are principle fiber bundles of $S_{\kappa_{1}, \kappa_{2}}^{3}$ with respective fibers $S_{\kappa_{1}}^{1}$ and $S_{\kappa_{1} \kappa_{2}}^{1}$ and they give the space of space-like and time-like geodesics of the spacetime $\Sigma_{\kappa_{1}, \kappa_{2}}$, as $H \rightsquigarrow 2 \mathbf{j}$ and $P \rightsquigarrow 2 \mathbf{k}$. Note however that the bases $S_{\kappa_{1}, \kappa_{2}}^{3} /\langle\mathbf{j}\rangle$ and $S_{\kappa_{1}, \kappa_{2}}^{3} /\langle\mathbf{k}\rangle$ are not given by $\Sigma_{\kappa_{1}, \kappa_{2}}$, as the fibers do not lie in the complex lines $A z_{1}+B z_{2}=0$ which have the complex structure of $\mathbb{C}_{\kappa_{2}}$, not of $\mathbb{C}_{\kappa_{1}}$ nor $\mathbb{C}_{\kappa_{1} \kappa_{2}}$.

Theorem 3. Let $H \rightsquigarrow 2 \mathbf{j}, P \rightsquigarrow 2 \mathbf{k}$, and $K \rightsquigarrow 2 \mathbf{i}$ denote the respective generators for time translations, space translations, and boosts of the kinematical algebra with commutators

$$
[K, H]=P, \quad[K, P]=-\kappa_{2} H, \quad \text { and } \quad[H, P]=\kappa_{1} K \text {. }
$$

We can construct principal fiber bundles

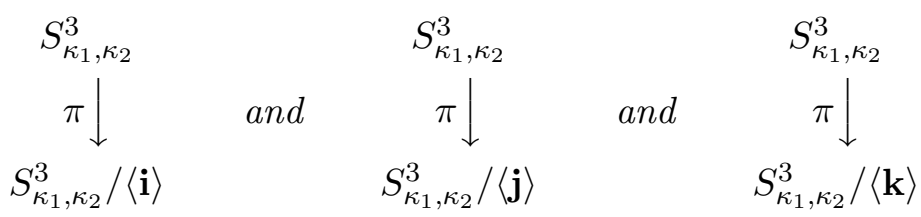

on the space $S_{\kappa_{1}, \kappa_{2}}^{3}$ of unit quaternions. Here the respective base spaces are the space of events, the space of space-like geodesics, and the space of time-like geodesics with corresponding Clifford flows on $S_{\kappa_{1}, \kappa_{2}}^{3}$ given by $\chi_{\mathbf{i}}(q)=\mathbf{i} q, \chi_{\mathbf{j}}(q)=\mathbf{j} q$, and $\chi_{\mathbf{k}}(q)=\mathbf{k} q$. The principal connections are determined by the distribution of horizontal planes spanned by $\{\mathbf{j} q, \mathbf{k} q\},\{\mathbf{i} q, \mathbf{k} q\}$, and $\{\mathbf{i} q, \mathbf{j} q\}$ with corresponding complex structures $\mathbb{C}_{\kappa_{2}}, \mathbb{C}_{\kappa_{1}}$, and $\mathbb{C}_{\kappa_{1} \kappa_{2}}$ for these planes.

\subsection{Optional reading on coordinate charts for $\Sigma_{\kappa_{1}, \kappa_{2}}$}

Complex lines will intersect $S_{\kappa_{1}, \kappa_{2}}^{3}$ exactly when $A z_{1}+B z_{2}=0$ and $z_{1} \bar{z}_{1}+\kappa_{1} z_{2} \bar{z}_{2}=1$. Let $\omega$ denote $\frac{1}{w}$. We can cover $\Sigma_{\kappa_{1}, \kappa_{2}}$ with two coordinate charts: Let $U_{1}$ denote the set of points $\left[\frac{B}{A}\right]$ of $\Sigma_{\kappa_{1}, \kappa_{2}}$ where $A$ is not a zero divisor, and $U_{2}$ the set of points $\left[\frac{A}{B}\right]$ where $B$ is not a zero divisor. Then the coordinate charts $\phi_{1}: U_{1} \rightarrow \mathbb{C}_{\kappa_{2}}$ and $\phi_{2}: U_{2} \rightarrow \mathbb{C}_{\kappa_{2}}$ are given by 
$\phi_{1}\left(\left[\frac{B}{A}\right]\right)=\omega=-\frac{z_{1}}{z_{2}} \in \mathbb{C}_{\kappa_{2}}$ and $\phi_{2}\left(\left[\frac{A}{B}\right]\right)=w=-\frac{z_{2}}{z_{1}} \in \mathbb{C}_{\kappa_{2}}$ respectively $^{8}$. If $S_{\kappa_{1}, \kappa_{2}}^{3} \stackrel{\pi}{\longrightarrow} \Sigma_{\kappa_{1}, \kappa_{2}}$ defines the projection map, then

$$
\left(\phi_{1} \circ \pi\right)^{-1}(\omega)=\left\{e^{\mathbf{i} \theta} \frac{1+\omega \mathbf{j}}{\sqrt{1+\kappa_{1}|\omega|^{2}}}\right\} \quad \text { and } \quad\left(\phi_{2} \circ \pi\right)^{-1}(w)=\left\{e^{\mathbf{i} \theta} \frac{w+\mathbf{j}}{\sqrt{|w|^{2}+\kappa_{1}}}\right\},
$$

where $e^{\mathbf{i} \theta}$ is an arbitrary element of $S_{\kappa_{2}}^{1}$. We can then give a product structure to $\pi^{-1}\left(U_{1}\right) \subset$ $S_{\kappa_{1}, \kappa_{2}}^{3}$ and to $\pi^{-1}\left(U_{2}\right) \subset S_{\kappa_{1}, \kappa_{2}}^{3}$ by

$$
\Phi_{1}\left(\omega, e^{\mathbf{i} \theta}\right)=e^{\mathbf{i} \theta} \frac{1+\omega \mathbf{j}}{\sqrt{1+\kappa_{1}|\omega|^{2}}} \quad \text { and } \quad \Phi_{2}\left(w, e^{\mathbf{i} \theta}\right)=e^{\mathbf{i} \theta} \frac{w+\mathbf{j}}{\sqrt{|w|^{2}+\kappa_{1}}}
$$

respectively. This trivializing cover of $S_{\kappa_{1}, \kappa_{2}}^{3}$ has a gluing map

$$
\Phi_{2}^{-1} \circ \Phi_{1}\left(\omega, e^{\mathbf{i} \theta}\right)=\left(\frac{1}{\omega}, e^{\mathbf{i} \theta} \frac{\omega}{|\omega|}\right) .
$$

We can also map $\Sigma_{\kappa_{1}, \kappa_{2}}$ to the unit sphere in $\mathbb{R}^{3}$ with metric ${ }^{9} d y^{2}+\kappa_{1} d t^{2}+\kappa_{1} \kappa_{2} d x^{2}$ by

$$
\omega=-\frac{z_{1}}{z_{2}} \mapsto\left(\mathcal{R} \frac{2 \omega}{1+\kappa_{1}|\omega|^{2}}, \mathcal{I} \frac{2 \omega}{1+\kappa_{1}|\omega|^{2}}, \frac{-1+\kappa_{1}|\omega|^{2}}{1+\kappa_{1}|\omega|^{2}}\right)
$$

or

$$
w=-\frac{z_{2}}{z_{1}} \mapsto\left(\mathcal{R} \frac{2 w}{1+\kappa_{1}|w|^{2}}, \mathcal{I} \frac{2 w}{1+\kappa_{1}|w|^{2}}, \frac{-1+\kappa_{1}|w|^{2}}{1+\kappa_{1}|w|^{2}}\right)
$$

as can be checked directly.

\section{The principal connection form}

The right invariant one-forms on $S_{\kappa_{1}, \kappa_{2}}^{3}$ are given by

$$
(d U) U^{-1}=\left(\begin{array}{cc}
d z_{1} & d z_{2} \\
-\kappa_{1} d \overline{z_{2}} & d \overline{z_{1}}
\end{array}\right)\left(\begin{array}{cc}
\overline{z_{1}} & -z_{2} \\
\kappa_{1} \overline{z_{2}} & z_{1}
\end{array}\right)=\left(\begin{array}{cc}
\overline{z_{1}} d z_{1}+\kappa_{1} \overline{z_{2}} d z_{2} & -z_{2} d z_{1}+z_{1} d z_{2} \\
-\kappa_{1} \overline{z_{1}} d \overline{z_{2}}+\kappa_{1} \overline{z_{2}} d \overline{z_{1}} & \kappa_{1} z_{2} d \overline{z_{2}}+z_{1} d \overline{z_{1}}
\end{array}\right)
$$

and the left invariant one-forms on $S_{\kappa_{1}, \kappa_{2}}^{3}$ are given by

$$
U^{-1} d U=\left(\begin{array}{cc}
\overline{z_{1}} & -z_{2} \\
\kappa_{1} \overline{z_{2}} & z_{1}
\end{array}\right)\left(\begin{array}{cc}
d z_{1} & d z_{2} \\
-\kappa_{1} d \overline{z_{2}} & d \overline{z_{1}}
\end{array}\right)=\left(\begin{array}{cc}
\overline{z_{1}} d z_{1}+\kappa_{1} z_{2} \overline{d z_{2}} & -z_{2} d \overline{z_{1}}+\overline{z_{1}} d z_{2} \\
-\kappa_{1} z_{1} d \overline{z_{2}}+\kappa_{1} \overline{z_{2}} d z_{1} & \kappa_{1} \overline{z_{2}} d z_{2}+z_{1} d \overline{z_{1}}
\end{array}\right) .
$$

We will show that the principal connection form is given by the right invariant form $\lambda=$ $\overline{z_{1}} d z_{1}+\kappa_{1} z_{2} \overline{d z_{2}}$. Let $J$ denote the almost complex structure on $\mathbb{R}^{4}$ that is compatible with multiplying $\left(z_{1}, z_{2}\right)$ by $i$ in $\mathbb{C}_{\kappa_{2}}^{2}$. Then

$$
\begin{aligned}
i\left(z_{1}, z_{2}\right)=\left(-\kappa_{2} y+i x,-\kappa_{2} v+i u\right) \\
\rightsquigarrow J X=J\left(\begin{array}{l}
x \\
y \\
u \\
v
\end{array}\right)=\left(\begin{array}{rr:rr}
0 & -\kappa_{2} & 0 & 0 \\
1 & 0 & 0 & 0 \\
\hdashline 0 & 0 & 0 & -\kappa_{2} \\
0 & 0 & 1 & 0
\end{array}\right)\left(\begin{array}{c}
x \\
y \\
u \\
v
\end{array}\right)=\left(\begin{array}{c}
-\kappa_{2} y \\
x \\
-\kappa_{2} v \\
u
\end{array}\right),
\end{aligned}
$$

\footnotetext{
${ }^{8}$ Note that the map $w \mapsto \omega=\frac{1}{w}=\frac{\bar{w}}{w \bar{w}}$ is conformal on the set of non-zero divisors.

${ }^{9}$ Recall that $S O_{\kappa_{1}, \kappa_{2}}(3)$ is the group of isometries of $\mathbb{R}^{3}=\{(y, t, x) \mid y, t, x \in \mathbb{R}\}$ with metric $d y^{2}+\kappa_{1} d t^{2}+$ $\kappa_{1} \kappa_{2} d x^{2}$.
} 
where $J^{2}=-\kappa_{2} I$ and $I$ is the identity matrix. Recall that the Hermitian inner product on $\mathbb{C}_{\kappa_{2}}^{2}$ is given by

$$
\begin{aligned}
\langle z, w\rangle & =z_{1} \overline{z_{2}}+\kappa_{1} w_{1} \overline{w_{2}} \\
& =\left(x_{1} x_{2}+\kappa_{2} y_{1} y_{2}+\kappa_{1} u_{1} u_{2}+\kappa_{1} \kappa_{2} v_{1} v_{2}\right)+i\left(y_{1} x_{2}-x_{1} y_{2}+\kappa_{1} v_{1} u_{2}-\kappa_{1} u_{1} v_{2}\right),
\end{aligned}
$$

where $z=\left(z_{1}, z_{2}\right)=\left(x_{1}+i y_{1}, u_{1}+i v_{1}\right)$ and $w=\left(w_{1}, w_{2}\right)=\left(x_{2}+i y_{2}, u_{2}+i v_{2}\right)$. In real coordinates we can write this as $\left\langle X_{1}, X_{2}\right\rangle=\left\langle\left\langle X_{1}, X_{2}\right\rangle\right\rangle+i \Phi\left(X_{1}, X_{2}\right)$, where $\left\langle\left\langle X_{1}, X_{2}\right\rangle\right\rangle$ and $\Phi\left(X_{1}, X_{2}\right)$ give the respective real and imaginary parts of the inner product $\left\langle X_{1}, X_{2}\right\rangle$, and where

$$
X_{1}=\left(\begin{array}{l}
x_{1} \\
y_{1} \\
u_{1} \\
v_{1}
\end{array}\right), \quad X_{2}=\left(\begin{array}{l}
x_{2} \\
y_{2} \\
u_{2} \\
v_{2}
\end{array}\right)
$$

So

$$
\left\langle\left\langle X_{1}, X_{2}\right\rangle\right\rangle=X_{1}^{T}\left(\begin{array}{cccc}
1 & 0 & 0 & 0 \\
0 & \kappa_{2} & 0 & 0 \\
0 & 0 & \kappa_{1} & 0 \\
0 & 0 & 0 & \kappa_{1} \kappa_{2}
\end{array}\right) X_{2}
$$

and

$$
\begin{aligned}
& \Phi\left(X_{1}, X_{2}\right)=-\frac{1}{\kappa_{2}}\left\langle\left\langle J X_{1}, X_{2}\right\rangle\right\rangle=\frac{1}{\kappa_{2}}\left\langle\left\langle X_{1}, J X_{2}\right\rangle\right\rangle \\
& =-\frac{1}{\kappa_{2}}\left(\begin{array}{llll}
-\kappa_{2} y_{1} & x_{1} & -\kappa_{2} v_{1} & u_{1}
\end{array}\right)\left(\begin{array}{cccc}
1 & 0 & 0 & 0 \\
0 & \kappa_{2} & 0 & 0 \\
0 & 0 & \kappa_{1} & 0 \\
0 & 0 & 0 & \kappa_{1} \kappa_{2}
\end{array}\right)\left(\begin{array}{l}
x_{2} \\
y_{2} \\
u_{2} \\
v_{2}
\end{array}\right) \\
& =-\frac{1}{\kappa_{2}} X_{1}^{T}\left(\begin{array}{cc:cc}
0 & 1 & 0 & 0 \\
-\kappa_{2} & 0 & 0 & 0 \\
\hdashline 0 & 0 & 0 & 1 \\
0 & 0 & -\kappa_{2} & 0
\end{array}\right)\left(\begin{array}{cccc}
1 & 0 & 0 & 0 \\
0 & \kappa_{2} & 0 & 0 \\
0 & 0 & \kappa_{1} & 0 \\
0 & 0 & 0 & \kappa_{1} \kappa_{2}
\end{array}\right) X_{2} \\
& =-\frac{1}{\kappa_{2}} X_{1}^{T}\left(\begin{array}{ccc:cc}
0 & \kappa_{2} & 0 & 0 \\
-\kappa_{2} & 0 & 0 & 0 \\
\hdashline 0 & 0 & 0 & \kappa_{1} \kappa_{2} \\
0 & 0 & -\kappa_{1} \kappa_{2} & 0
\end{array}\right) X_{2} \\
& =X_{1}^{T}\left(\begin{array}{cc:cc}
0 & -1 & 0 & 0 \\
1 & 0 & 0 & 0 \\
\hdashline 0 & 0 & 0 & -\kappa_{1} \\
0 & 0 & \kappa_{1} & 0
\end{array}\right) X_{2} \text {. }
\end{aligned}
$$


If we let this last matrix describe a (possible degenerate) symplectic form $\varpi$, then $\left\langle\left\langle X_{1}, J X_{2}\right\rangle\right\rangle=$ $\kappa_{2} \varpi\left(X_{1}, X_{2}\right)$, so that $\varpi$ is compatible with $J$. In the degenerate case when $\kappa_{2}=0$ and the above calculations for $\Phi\left(X_{1}, X_{2}\right)$ make no sense, we can write $\kappa_{2} \Phi\left(X_{1}, X_{2}\right)=\left\langle\left\langle J X_{1}, X_{2}\right\rangle\right\rangle$ and we then rescale ${ }^{10}$ by canceling out the factor of $\kappa_{2}$ on both the left and the right sides of this equation.

The unit sphere $S_{\kappa_{1}, \kappa_{2}}^{3}$ in $\mathbb{R}^{4}$ can be described by the equation $\langle\langle X, X\rangle\rangle=1$. The orbit of $\left(z_{1}, z_{2}\right)$ in $S_{\kappa_{1}, \kappa_{2}}^{3}$ under the $S_{\kappa_{2}}^{1}$ action that is given by

$$
\begin{aligned}
\left(z_{1}, z_{2}\right) \mapsto & e^{i \theta}\left(z_{1}, z_{2}\right)=\left(C_{\kappa_{2}}(\theta)+i S_{\kappa_{2}}(\theta)\right)(x+i y, u+i v) \\
= & {\left[C_{\kappa_{2}}(\theta) x-\kappa_{2} S_{\kappa_{2}}(\theta) y+i\left(C_{\kappa_{2}}(\theta) y+S_{\kappa_{2}}(\theta) x\right),\right.} \\
& \left.C_{\kappa_{2}}(\theta) u-\kappa_{2} S_{\kappa_{2}}(\theta) v+i\left(C_{\kappa_{2}}(\theta) v+S_{\kappa_{2}}(\theta) u\right)\right]
\end{aligned}
$$

is, in real terms, given by $X \mapsto X C_{\kappa_{2}}(\theta)+J X S_{\kappa_{2}}(\theta)$. Since $X$ and $J X$ are orthogonal ${ }^{11}$, a unit circle is traced out in the plane of $\mathbb{R}^{4}$ that contains both $X$ and $J X$ : Recall that $C^{2}(\theta)+$ $\kappa_{2} S^{2}(\theta)=1$, noting that $\langle\langle J X, J X\rangle\rangle=\kappa_{2}$ as can be calculated directly taking into account the fact that $\langle\langle X, X\rangle\rangle=1$. This calculation also shows that all fibers are of the same size.

The vector tangent to the fiber through $\left(z_{1}, z_{2}\right)$ is given by $i\left(z_{1}, z_{2}\right)$ or, in real terms, by $J X$. If $X(t)$ is a differentiable curve lying in $S_{\kappa_{1}, \kappa_{2}}^{3}$, then $|X(t)|^{2}=1$ implies that $\langle\langle X(t), \dot{X}(t)\rangle\rangle=0$. If this curve is also orthogonal to the fiber passing through $X(t)$, then we must also have that $\langle\langle J X(t), \dot{X}(t)\rangle\rangle=0$. So $\lambda=\bar{z} d z=\overline{z_{1}} d z_{1}+\kappa_{1} \overline{z_{2}} d z_{2}$ is the principal connection form as $\lambda$ is clearly equivariant.

The curvature form is $d \lambda=d \overline{z_{1}} \wedge d z_{1}+\kappa_{1} d \overline{z_{2}} \wedge d z_{2}$. Now if $X$ and $Y$ are curves on $S_{\kappa_{1}, \kappa_{2}}^{3}$ so that $\dot{X}=\mathbf{j} q$ and $\dot{Y}=\mathbf{k} q$ at a point $q \in S_{\kappa_{1}, \kappa_{2}}^{3}$, then $d \lambda(\dot{X}, \dot{Y})=\langle\dot{X}, \dot{Y}\rangle=\mathbf{j} q \overline{\mathbf{k} q}=-\mathbf{j} q \bar{q} \mathbf{k}=-\kappa_{1} \mathbf{i}$. Also, recall that $[\mathbf{j}, \mathbf{k}]=2 \kappa_{1} \mathbf{i}$. And so the curvature is given by $\kappa_{1}$ and $\lambda$ is a contact form exactly when $\kappa_{1} \neq 0$. The area of the infinitesimal rectangle $D$ defined by $\epsilon \mathbf{j} q$ and $\delta \mathbf{k} q$ has area $\epsilon \delta$ and the holonomy $\theta$ around the rectangle is given by $\epsilon \delta \kappa_{1}$ : So the curvature is defined by $\theta=\int_{D} K d A$ or $\epsilon \delta \kappa_{1}=\epsilon \delta K$ so that $K=\kappa_{1}$. Our definition of area is in lieu of rescaling metrics: See [6] or [7] for different calculations of the the curvature. In effect we are treating $\{\mathbf{i} q, \mathbf{j} q, \mathbf{k} q\}$ as an orthonormal frame.

Finally we will use the principal connection form to derive the metric of the spacetime $\Sigma_{\kappa_{1}, \kappa_{2}}$ (see also [8]): Recall that the metric is to be rescaled by dividing by $\kappa_{1}$. We define a horizontal curve $X(t)$ (so $\bar{X}(t) \dot{X}(t)=0$ ) passing through $q=z_{1}+z_{2} \mathbf{j}$ in $S_{\kappa_{1}, \kappa_{2}}^{3}$ as follows

$$
X(t)=\left(C_{\kappa_{1}}(t)+S_{\kappa_{1}}(t) \mathbf{j}\right)\left(z_{1}+z_{2} \mathbf{j}\right)=\left(C_{\kappa_{1}}(t) z_{1}-\kappa_{1} S_{\kappa_{1}}(t) \overline{z_{2}}\right)+\left(C_{\kappa_{1}}(t) z_{2}+S_{\kappa_{1}}(t) \overline{z_{1}}\right) \mathbf{j} .
$$

Then

$$
\begin{aligned}
& \dot{X}(t)=\left(-\kappa_{1} S_{\kappa_{1}}(t) z_{1}-\kappa_{1} C_{\kappa_{1}}(t) \overline{z_{2}}\right)+\left(-\kappa_{1} S_{\kappa_{1}}(t) z_{2}+C_{\kappa_{1}}(t) \overline{z_{1}}\right) \mathbf{j}, \\
& \dot{X}(0)=-\kappa_{1} \overline{z_{2}}+\overline{z_{1}} \mathbf{j}, \\
& |\bar{X}(0)|^{2}=\kappa_{1}{ }^{2}\left|z_{2}\right|^{2}+\kappa_{1}\left|z_{1}\right|^{2}=1 .
\end{aligned}
$$

Now $X(t)$ is the horizontal lift of the curve $w(t)$ in $\Sigma_{\kappa_{1}, \kappa_{2}}$ where

$$
\begin{aligned}
& w(t)=\frac{C_{\kappa_{1}}(t) z_{2}+S_{\kappa_{1}}(t) \overline{z_{1}}}{C_{\kappa_{1}}(t) z_{1}-\kappa_{1} S_{\kappa_{1}}(t) \overline{z_{2}}}, \\
& w(0)=\frac{z_{2}}{z_{1}}=w,
\end{aligned}
$$

\footnotetext{
${ }^{10}$ Similarly we rescaled by dividing by $\kappa_{1}$ or $\kappa_{2}$, even if they were equal to zero, in order to obtain the metrics on the homogeneous spaces $S_{\kappa_{1}, \kappa_{2}}^{3} /\langle\mathbf{i}\rangle, S_{\kappa_{1}, \kappa_{2}}^{3} /\langle\mathbf{j}\rangle$, and $S_{\kappa_{1}, \kappa_{2}}^{3} /\langle\mathbf{k}\rangle$.

${ }^{11}$ Neither $X$ nor $J X$ is the zero vector, and we also have that $\langle\langle X, J X\rangle\rangle=\mathcal{R}(\langle X, J X\rangle)=\mathcal{R}(-i)=0$.
} 


$$
\begin{aligned}
\dot{w}(t)= & \left(-\kappa_{1} S_{\kappa_{1}}(t) z_{2}+C_{\kappa_{1}}(t) \overline{z_{1}}\right)\left(C_{\kappa_{1}}(t) z_{1}-\kappa_{1} S_{\kappa_{1}}(t) \overline{z_{2}}\right)-\left(C_{\kappa_{1}}(t) z_{2}+S_{\kappa_{1}}(t) \overline{z_{1}}\right) \\
& \times\left(-\kappa_{1} S_{\kappa_{1}}(t) z_{1}-\kappa_{1} C_{\kappa_{1}}(t) \overline{z_{2}}\right)\left(C_{\kappa_{1}}(t) z_{1}-\kappa_{1} S_{\kappa_{1}}(t) \overline{z_{2}}\right)^{-2}, \\
\dot{w}(0)= & \frac{\overline{z_{1}} z_{1}+\kappa_{1} z_{2} \overline{z_{2}}}{z_{1}^{2}}=\frac{1}{z_{1}^{2}} .
\end{aligned}
$$

Since

$$
\left|z_{1}\right|^{2}\left(1+\kappa_{1}|w|^{2}\right)=\left(1+\frac{\kappa_{1}\left|z_{2}\right|^{2}}{\left|z_{1}\right|^{2}}\right)\left|z_{1}\right|^{2}=\left|z_{1}\right|^{2}+\kappa_{1}\left|z_{2}\right|^{2}=1,
$$

then $\left|z_{1}\right|^{2}=\frac{1}{\left(1+\kappa_{1}|w|^{2}\right)}$. As $|\mathbf{j} q|^{2}=\kappa_{1}$ and $|\mathbf{k} q|^{2}=\kappa_{1} \kappa_{2}$, the metric $\frac{1}{\kappa_{1}} d s^{2}$ induced on the base space is given by

$$
z_{1}^{2} \bar{z}_{1}^{2} d w d \bar{w}=\frac{d w d \bar{w}}{\left(1+\kappa_{1}|w|^{2}\right)^{2}} .
$$

Theorem 4. Let $H \rightsquigarrow 2 \mathbf{j}, P \rightsquigarrow 2 \mathbf{k}$, and $K \rightsquigarrow 2 \mathbf{i}$ denote the respective generators for time translations, space translations, and boosts of the kinematical algebra with commutators

$$
[K, H]=P, \quad[K, P]=-\kappa_{2} H, \quad \text { and } \quad[H, P]=\kappa_{1} K .
$$

Then the principal fiber bundle

$$
\begin{gathered}
S_{\kappa_{1}, \kappa_{2}}^{3} \\
\pi \downarrow \\
S_{\kappa_{1}, \kappa_{2}}^{3} /\langle\mathbf{i}\rangle
\end{gathered}
$$

has $\lambda=\overline{z_{1}} d z_{1}+\kappa_{1} z_{2} \overline{d z_{2}}$ as its principal connection form. The base space, which is the space of events, has induced metric

$$
d s^{2}=\frac{d w d \bar{w}}{\left(1+\kappa_{1}|w|^{2}\right)^{2}}
$$

and constant curvature $\kappa_{1}$.

In conclusion, it is hoped that the aims of this paper were met, that the nice structure of the Hopf fibration $S^{3} \longrightarrow S^{2}$ was generalized in an appealing way, not only for the classical Riemannian surfaces of constant curvature, but especially for the study of $(1+1)$ kinematics. It is also hoped that the reader will find that these fibrations give a new perspective on these simple kinematical structures. Finally, I wish to thank the reviewers for their many helpful suggestions on how this paper could be improved.

\section{References}

[1] Bacry H., Levy-Leblond J.-M., Possible kinematics, J. Math. Phys. 9 (1967), 1605-1614.

[2] Ballesteros A., Herranz F.J., Superintegrability on three-dimensional Riemannian and relativistic spaces of constant curvature, SIGMA 2 (2006), 010, 22 pages, math-ph/0512084.

[3] Harkin A.A., Harkin J.B., Geometry of generalized complex numbers, Math. Mag. 77 (2004), 118-129.

[4] Herranz F.J., Ortega R., Santander M., Trigonometry of spacetimes: a new self-dual approach to a curvature/signature (in)dependent trigonometry, J. Phys. A: Math. Gen. 33 (2000), 4525-4551, math-ph/9910041. 
[5] Herranz F.J., Santander M., Homogeneous phase spaces: the Cayley-Klein framerwork, in Geometría y Física (Madrid, Real Academia de Ciencias, 1996), Editors J.F. Cariñena et al., Mem. Real Acad. Cienc. Exact. Fis. Natur. Madrid 32 (1998), 59-84, physics/9702030.

[6] Herranz F.J., Santander M., Conformal symmetries of spacetimes, J. Phys. A: Math. Gen. 35 (2002), 66016618, math-ph/0110019.

[7] McRae A.S., The Gauss-Bonnet theorem for Cayley-Klein geometries of dimension two, New York J. Math. 12 (2006), 143-155.

[8] McRae A.S., Clifford algebras and possible kinematics, SIGMA 3 (2007), 079, 29 pages, arXiv:0707.2869.

[9] Penrose R., The road to reality. A complete guide to the laws of the universe, Alfred A. Knopf, Inc., New York, 2005.

[10] Urbantke H.K., The Hopf fibration - seven times in physics, J. Geom. Phys. 46 (2003), 125-150.

[11] Van der Waerden B.L., Algebra, Vol. 2, Springer-Verlag, New York, 1991.

[12] Yaglom I.M., A simple non-Euclidean geometry and its physical basis: an elementary account of Galilean geometry and the Galilean principle of relativity, Heidelberg Science Library, Translated from the Russian by Abe Shenitzer, With the editorial assistance of Basil Gordon, Springer-Verlag, New York - Heidelberg, 1979. 